\title{
The interprofessional collaboration between nurses and speech-language pathologists working with patients diagnosed with dysphagia in skilled nursing facilities
}

\author{
Kaitlin Dondorf ${ }^{1}$, Renee Fabus ${ }^{* 1}$, Akhtar Ebrahimi Ghassemi ${ }^{2}$ \\ ${ }^{1}$ Department of Communication Sciences \& Disorders, Adelphi University, Garden City, United States \\ ${ }^{2}$ Mental Health, Family \& Community Systems Department, College of Nursing \& Public Health, Adelphi University, Garden \\ City, United States
}

Received: July 20, 2015

Accepted: November 15, $2015 \quad$ Online Published: December 7, 2015

DOI: $10.5430 /$ jnep.v6n4p17

URL: http://dx.doi.org/10.5430/jnep.v6n4p17

\begin{abstract}
The speech-language pathologist (SLP) is the primary person responsible for the assessment and intervention of individuals with swallowing disorders. In skilled nursing facilities, both nurses and SLPs work closely with patients diagnosed with strokes. Aspiration pneumonia is the most common cause of death in patients diagnosed with dysphagia resulting from a stroke. Due to the large number of patients with dysphagia in healthcare facilities, it is pertinent that SLP and nurses collaborate during clinical practice to improve patient outcomes. This is a discussion paper emphasizing the importance of interprofessional collaboration. Due to increasing complexity of patient care, it is important to establish collaborations early in interdisciplinary healthcare training in order to improve quality of patient care. The interdisciplinary collaboration should become a standard for training healthcare professionals including nurses and speech-language pathologists in today's complex healthcare system.
\end{abstract}

Key Words: Speech-language pathologist, Stroke, Interprofessional collaboration, Skilled nursing facility

\section{INTRODUCTION}

Dysphagia, or impaired swallowing, is a common health concern amongst residents in skilled nursing facilities (SNF). Individuals presenting with anatomical or physiological deficits in the oral cavity, pharynx, larynx or esophagus may exhibit signs of dysphagia. ${ }^{[1]}$ In the United States, approximately 600,000 patients with neurological impairment are affected by dysphagia each year. ${ }^{[2]}$ The Speech-Language Pathologist (SLP) is the primary professional responsible for the evaluation and treatment of swallowing disorders in a healthcare setting and dysphagia assessment and treatment will account for the majority of the SLPs caseload in a SNF. Dysphagia may have several detrimental effects, particularly in the elderly population, such as aspiration pneumonia, dehydration, malnutrition, decreased pulmonary function, or even death. ${ }^{[3]}$ According to Threats, the use of the World Health Organization's International Classification of Functioning, Disability and Health (ICF) by SLPs can expand and enhance the outcomes for individuals with dysphagia. The World Health Organization (WHO) defines health as "the complete physical, mental, and social well-being and not merely the absence of disease or infirmity". ${ }^{[4]}$ Viewing dysphagia through this lens allows us to see that a swallowing disorder is not simply a medical issue, but a sequela of a disease that may lead to

*Correspondence: Renee Fabus; Email: fabus@adelphi.edu; Address: Department of Communication Sciences \& Disorders, Adelphi University, Garden City, United States. 
multiple limitations in the participation of daily activities, as well as isolation and depression.

The WHO also urges SLPs to educate and collaborate with other disciplines to better understand and manage dysphagia and appreciate the multifaceted impact of this disorder. The SLP uses a combination of clinical expertise, knowledge, experience and evidence-based practice along with collaboration with other disciplines when working with patients with swallowing disorders. The SLP works together with physicians, dieticians, occupational therapists, physical therapists, respiratory therapists, families and especially nurses when working with a patient with dysphagia. An interdisciplinary approach to the assessment and management of dysphagia is essential in providing best patient care. This paper highlights the importance of interprofessional collaboration between nurses and SLPs.

The WHO recognizes collaboration amongst professionals in education and practice as an "innovative strategy that will play an important role in mitigating the global health workforce crisis". ${ }^{[5]}$ Interprofessional collaboration involves individuals from two or more professions coming together to learn from and with each other in order to work as a team and improve patient health outcomes. This collaborative practice also involves working closely with patients, families, caregivers, and communities to provide the highest level of patient care. By engaging with professionals from a different expertise and background, SLPs can broaden their knowledge and practice to achieve specific health goals for patients with dysphagia. At this time, the healthcare world, and SLPs particularly, are experiencing staff shortages at their place of employment, may have very large caseloads, and are working hard to balance the multiple responsibilities of service delivery, such as family education, and documentation and billing of dysphagia services. The Framework for Action on Interprofessional Education and Collaborative Practice ${ }^{[5]}$ provides guidelines for creating successful collaborative teams, and outlines ways to apply this collaborative process in different settings. The aim of this article is to discuss interprofessional collaboration with SLPs and nurses within an SNF using the WHO guidelines in order to strengthen health system performance and improve health outcomes for patients with dysphagia.

\section{The COLlaborative RELATIONSHIP BE- TWEEN NURSES AND SPEECH-LANGUAGE PATHOLOGISTS}

A partnership between an SLP and a Nurse is essential in the assessment and management of dysphagia in an SNF. Nursing observations and opinions are invaluable in fully understanding all dimensions of a case for a patient with dysphagia. Nurses are often the eyes and ears for the SLP who may only see a patient for a short treatment session per day. Having open communication between nurses and SLPs can make a huge difference in the quality of life of an individual with a swallowing disorder. For example, Mr. $\mathrm{X}$ is coughing while taking medication this morning and reports "It is hard for me to swallow liquids!" The nurse can notify the SLP for a possible re-assessment of swallow function. Mr. X's swallow can then be re-evaluated and treated accordingly for improved swallow safety.

According to the WHO, the collaboration amongst disciplines will result in improved coordination of health services, appropriate referral to specialist resources, improved health outcomes for patients with chronic diseases, and overall improved patient safety and care. ${ }^{[5]}$ Collaborative practices can decrease patient's length of stay in a facility, reduce patient's medical complications, as well as reduce staff turnover, tensions and conflict among caregivers, and most importantly, reduce mortality rates. ${ }^{[5]}$ The goal to encouraging more integrated work relationships is to maximize limited health resources as well as to promote patient safety. The collaboration of nursing and SLP specifically targets the latter category. As stated earlier, dysphagia can lead to numerous medical issues, or even death. Often times, the simple communication between the two disciplines can greatly change the course of a patient's medical recovery or even their life.

\section{THE ROLE OF THE NURSING STAFF IN THE ASSESSMENT PROCESS}

Dysphagia screening and management are key processes in the care of patients post stroke. However, there is currently no agreement regarding the best approach to dysphagia screening. Dispute exists regarding what tool should be used for screening and who should administer the screening (e.g., nurse, speech-language pathologist). ${ }^{[6]}$ In a quantitative systematic review, "a formal dysphagia screening protocol (consisting of Burke water swallow test or variant developed de novo by the site) was statistically significantly associated with a reduced incidence of aspiration pneumonia in stroke survivors". ${ }^{[2]}$ The author's findings indicated that dysphagia-screening protocol used by nurses should include an algorithm for referral to speech language professionals for formal assessment. In one study, the use of the National Institute of Health Stroke Scale (NIHSS) was more apt in predicting dysphagia as compared to the Nursing Admission Dysphagia Screening Tool. The researchers suggested the use of NIHSS as an early screening tool for dysphagia among stroke patients. ${ }^{[7]}$ As suggested by Bravata, ${ }^{[7]}$ nurses can use both dysphagia screening tools (NIHSS and/or Nurs- 
ing Admission Dysphagia Screening Tool) as well as SLP consultation (the gold standard). Patients with swallowing difficulty should be evaluated using formal dysphagia screening protocols within 24 hours of admission and nurses should be trained to perform dysphagia screening to safeguard the accuracy of implementing the procedure. ${ }^{[2,6]}$

Several researchers have studied the benefits of training nurses in screening for dysphagia. ${ }^{[8-11]}$ In some facilities, referrals to the SLP are made following a nurse's screen that determines the need for further dysphagia evaluation. Various researchers have created different protocols for a nurse to follow in order to conduct a dysphagia screen, therefore, there is not one universally utilized swallow screen amongst medical facilities, nor are all facilities utilizing nursing dysphagia screens. In one study, the SLPs trained the nurses in a medical facility to perform a swallow screen based on a specific tool created by the investigators. The SLPs trained the nurses on how to perform the screen effectively, including what signs and symptoms to observe that may possibly indicate a swallowing disorder. Results of the screen would be inputted into a computer system and automatically transferred to the SLP department for referral, as needed. The authors note that the program takes the commitment of nurses and SLPs and a true partnership for it to be successful. However, authors note that with success this type of program can result in early detection of dysphagia as it prompts a full assessment by a trained professional in dysphagia management, the SLP. SLPs must consider the value of the nurses' holistic view of the patient, their accessibility to caregivers, and their ability to repeat a screen at any time of day at the patient's necessity. ${ }^{[8]}$ Additionally, these authors agree with the research findings indicating that understanding and appreciating professional roles and responsibilities and communicating effectively are considered to be two core competencies for patient-centered collaborative practice. ${ }^{[12]}$

It should be noted that other researchers discuss a lack of education and clinical knowledge in the area of dysphagia, which may create hesitation amongst nurses in performing such a task. ${ }^{[9,10]} \mathrm{McHale}^{[10]}$ discusses expert nursing knowledge in the care of patients with dysphagia and reports the lack of inclusion of dysphagia information in basic nursing courses, as well as in nursing textbooks and a paucity of information as to the nurses' responsibilities when it relates to the assessment and management of dysphagia. This is where collaborative practice can play a major role in improving patient care. The $\mathrm{WHO}^{[5]}$ suggests certain strategies that may change the way health workers interact with one another to deliver care. For example, interdisciplinary education, such as in-services (education sessions and/or professional development), using evidence-based information as well as case studies, will increase knowledge and staff comfort level in working with patients with dysphagia. The $\mathrm{WHO}^{[5]}$ lists examples of mechanisms that can shape interprofessional education at the practice level: staff training, managerial commitment, institutional support, and learning outcomes. When it comes to dysphagia collaboration, the SLP can provide in-services for nursing staff and offer weekly or monthly meetings scheduled with management, including the SLP supervisors and nurse managers. In addition, both professionals should promote a collaborative approach, reach out to administration for support for multi-departmental educational opportunities, and develop clear and collaborative learning outcomes to include the time, focus and productivity of all involved.

\section{THE ROLE OF THE NURSING STAFF IN THE TREATMENT PROCESS}

Early detection and initiation of proper interventions may diminish complications related to dysphagia and improve quality of referrals, health outcomes, and quality of life for individuals with dysphagia. ${ }^{[2]}$ In acute settings, nurses are available on a 24-hour basis, and may distinguish individuals with dysphagia and begin interventions that may prevent further complications until SLPs can perform a formal assessment. Collaboration between nurses and SLPs should also occur during the treatment process when working with individuals with dysphagia. SLPs formulate a treatment plan based on the evaluation and instrumental measures. Afterwards, the SLP makes recommendations including the safest diet consistencies, postural changes, specific swallow maneuvers, oral hygiene, as well as safe swallow strategies that must be implemented in order for the individual to swallow safely. Nurses, as well as certified nursing assistants (CNA), play an important role in helping to implement the recommendations of the SLP. Following a dysphagia evaluation (often including instrumental assessment) the SLP will communicate the results of the evaluation to the nurse, as well as the necessary recommendations. For example, Mr. X had a Modified Barium Swallow Study completed today in which he was able to tolerate thin liquids with use of the chin-tuck posture. The SLP would communicate to the Nurse and CNAs that Mr. X must use the chin-tuck position whenever consuming thin liquids. The SLP may also add that Mr. X should only take small sips and not use straws. As mentioned earlier, the SLP may only see the patient for one short treatment session a day, while the nurse and CNA are in frequent contact with the patient. The CNA may observe or feed the patient during mealtime and assist in implementing the SLPs recommendations. Pelletier's (2004) research findings revealed strong evidence from three data collection meth- 
ods that CNAs' knowledge of dysphagia and feeding was inadequate in terms of technical skills, safety, and communication and noted that education and supervision are required in order to improve CNA feeding skills and knowledge. One particular result of Pelletier's study shows the importance of collaborative discussion among nursing home personnel and SLPs in ensuring that in-service content is pertinent to CNAs. Nurses and CNAs play a pivotal role in supervising and ensuring that patients' dysphagia needs (including oral hygiene, hydration, and nutritional requirements) are met in a holistic and safe manner. ${ }^{[13]}$

McCullough, Estes, McCullough and Rainey ${ }^{[14]}$ examined nurses' self-reported levels of compliance with SLPs' recommendations for safe feeding and swallowing techniques in caring for adults with dysphagia. It was found that out of 77 responses, nurses found their compliance with SLP recommendations to be high. However, it was also noted that approximately $80 \%$ of nurses voiced a need for more education regarding dysphagia management. This research emphasizes the importance of interprofessional collaboration between SLPs and nurses in the treatment of individuals with dysphagia.

Interprofessional learning can be used in nursing education and to foster an interdisciplinary approach to improve health outcomes, client-centered care, and quality of care. It would provide nursing and allied health students with opportunities to develop necessary skills to work with different health professionals in a collaborative manner.

\section{SUMMARY/CONCLUSIONS}

The ability to work with professionals from other disciplines to deliver collaborative, holistic, patient-centered care is considered a critical component of professional practice demanding a specific set of competencies. However, a commonly accepted framework for collaborative competencies is lacking, which makes consistent preparation of students and staff challenging. "By shifting the way health workers think about and interact with one another, the culture of the working environment and attitudes of the workforce will change, improving the working experience of staff and benefiting the community as a whole".[15] As emphasized in this paper, there are many ways that nurses and SLPs can work together to deliver collaborative care to patients with dysphagia and improve not only swallow function, but also, overall quality of life. As discussed, the WHO guidelines provide SLPs and nurses with the framework to strengthen our collaboration; helping us to work together to provide the highest quality patient care for individuals with dysphagia.

\section{REFERENCES}

[1] Sura L, Madhavan A, Carnaby G, et al. Dysphagia in the elderly: management and nutritional considerations. Clin Interv Aging. 2012; 7: 287-98. PMid:22956864

[2] Hines S, Wallace K, Crowe L, et al. Identification and nursing management of dysphagia in individuals with acute neurological impairment (update). Int J of Evid Based Healthc. 2011 Jun; 9(2): 148150. PMid:21599843 http://dx.doi.org/10.1111/j.1744-1 $609.2011 .00211 . x$

[3] Threats TT. Use of the ICF in dysphagia management. Semin Speech Lang. 2007 Nov; 28(4): 323-33. PMid:17935017 http: //dx.doi.org/10.1055/s-2007-986529

[4] World Health Organization. Constitution of the World Health Organization. 2006. Available from: http://www . who.int/governan ce/eb/who_constitution_en.pdf

[5] World Health Organization. Framework for action on interprofessional education \& collaborative practice. Geneva: World Health Organization.

[6] Hinchey JA, Shephard T, Furie K, et al. Stroke Practice Improvement Network Investigators. Formal dysphagia screening protocols prevent pneumonia. Stroke. 2005 Sep; 36(9): 1972-1976. PMid:16109909 ht tp://dx.doi.org/10.1161/01.STR.0000177529.86868.8d

[7] Bravata DM, Daggett VS, Woodword-Hagg H, et al. Comparison of two approaches to screenfor dysphagia among acute ischemic stroke patients: nursing admission screening tool versus National Institute of Health stroke scale. J Rehabil Res Dev. 2009; 46(9): 1127-34. http://dx.doi.org/10.1682/JRRD. 2008.12.0169

[8] Heritage M. A collaborative approach to the assessment and management of dysphagia. Int J Lang Commun Disord. 2001; 36(Suppl.):
369-74. PMid:11340814 http://dx.doi.org/10.3109/13682 820109177913

[9] Magnus V. Dysphagia training for nurses in an acute hospital settinga pragmatic approach. Int J Lang Commun Disord. 2001; 36(Suppl.): 375-8. PMid:11340815 http://dx.doi.org/10.3109/1368282 0109177914

[10] McHale JM, Phipps MA, Horvath K, et al. Expert nursing knowledge in the care of patients at risk of impaired swallowing. Image J Nurs Sch. 1998; 30(2): 137-41. http://dx.doi.org/10.1111/j.154 7-5069.1998.tb01268.x

[11] Sandhaus S, Zalon ML, Valenti D, et al. Promoting evidence-based dysphagia assessment and management by nurses. J Gerontol Nurs. 2009 Jun; 35(6): 20-7. PMid:19537291 http://dx.doi .org/10. 3928/00989134-20090331-08

[12] Suter E, Arndt J, Arthur N, et al. Role understanding and effective communication as core competencies for collaborative practice. J Interprof Care. 2009 Jan; 23(1): 45-51. PMid:19142782 http://dx.doi.org/10.1080/13561820802338579

[13] Hughes SM. Management of dysphagia in stroke patients. Nurs Older People. 2011 Apr; 23(3): 21-4.

[14] McCullough KC, Estes JL, McCullough GH, et al. RN compliance with SLP dysphagia recommendations in acute care. Topics in Geriatric Rehabilitation. 2007 Oct; 23(4): 330-340. http: //dx.doi.org/10.1097/01.TGR.0000299161.44869.26

[15] Pelletier CA. What do certified nursing assistants actually know about dysphagia and feeding nursing home residents? Am J Speech Lang Pathol. 2004 May; 13(2): 99-113. http://dx.doi.org/10.1044 /1058-0360(2004/012) 